\title{
Position-dependent noncommutative quantum models: Exact solution of the harmonic oscillator
}

\author{
Dine Ousmane Samary \\ Perimeter Institute for Theoretical Physics \\ 31 Caroline St. N. Waterloo, ON N2L 2Y5, Canada \\ International Chair in Mathematical Physics and Applications \\ (ICMPA-UNESCO Chair), University of Abomey-Calavi, \\ 072B.P.50, Cotonou, Rep. of Benin \\ E-mail: ousmanesamarydine@yahoo.fr
}

\begin{abstract}
This paper is devoted to find the exact solution of the harmonic oscillator in a position-dependent 4-dimensional noncommutative phase space. The noncommutative phase space that we consider is described by the commutation relations between coordinates and momenta: $\left[\hat{x}^{1}, \hat{x}^{2}\right]=i \theta\left(1+\omega_{2} \hat{x}^{2}\right),\left[\hat{p}^{1}, \hat{p}^{2}\right]=i \bar{\theta},\left[\hat{x}^{i}, \hat{p}^{j}\right]=i \hbar_{e f f} \delta^{i j}$. We give an analytical method to solve the eigenvalue problem of the harmonic oscillator within this deformation algebra.
\end{abstract}

Key words: Noncommutative phase space, Moyal star product, eigenvalues problem, harmonic oscillator.

\section{Introduction}

Noncommutative (NC) geometry plays an increasing role in the search of a unifying theory of gravity and quantum mechanics and is a framework built for understanding physics at short distances. Within this framework, the past two decades have witnessed important progresses toward the solution of various quantum models, in particular, the harmonic oscillator in NC spaces. There exists a large number of papers which address this class of problem. We will focus on the most recent developments discussing particular tractable models and specific ways to realize $\mathrm{NC}$ spaces called Moyal spaces [1]-[22].

The Moyal type NC space is a concrete proposal for a space where the coordinate operators $\hat{x}^{\mu}$ satisfy the commutation relation

$$
\left[\hat{x}^{\mu}, \hat{x}^{\nu}\right]=i \theta^{\mu \nu}
$$

and where $\theta^{\mu \nu}$ is an antisymmetric tensor of space dimension (length $)^{2}$. The noncommutativity specified by (11) can be as well realized in terms of a star product. In this point of view, the ordinary multiplication of functions is replaced by the Moyal star product defined for $f, g \in C^{\infty}\left(\mathbb{R}^{D}\right)$ by

$$
(f \star g)(x)=\mathbf{m}\left[\exp \left(\frac{i}{2} \theta^{\mu \nu} \partial_{\mu} \otimes \partial_{\nu}\right)(f \otimes g)(x)\right], \quad \mathbf{m}(f \otimes g)(x)=f(x) \cdot g(x) .
$$


Then the commutation relation (1) becomes

$$
\left[x^{\mu}, x^{\nu}\right]_{\star}=x^{\mu} \star x^{\nu}-x^{\nu} \star x^{\mu}=i \theta^{\mu \nu}
$$

with now commuting coordinates $x^{\mu}$. The noncommutativity of space coordinates can be naturally incorporated into the quantum field theory framework. Subsequently, NC field theories and quantum mechanics have studied extensively [2]. There is however a more general structure extending the Moyal brackets (3). Consider that one replaces the commutation relation (1) by following [6]-[7]

$$
\left[\hat{x}^{\mu}, \hat{x}^{\nu}\right]=i \theta^{\mu \nu} e(\hat{x})
$$

where $e(\hat{x})$ is an arbitrary dimensionless function which depends on the coordinates. The same space can be again realized using another star product called the twisted Moyal product generalizing (22). Taking $e(x)$ positive, the star product

$$
(f \star g)(x)=\mathbf{m}\left[\exp \left(\frac{i}{2} \theta^{\mu \nu} \sqrt{e(x)} \partial_{\mu} \otimes \sqrt{e(x)} \partial_{\nu}\right)(f \otimes g)(x)\right]
$$

can be used to generate

$$
\left[x^{\mu}, x^{\nu}\right]_{\star}=i \theta^{\mu \nu} e(x)
$$

now extending (3). The choice of the function $e(x)$ depends on the physical considerations which may encode minimal length [4] or the integrability of some dynamical Hamiltonians [8].

We emphasize the fact that a necessary condition for having an associative star product from (5) is given by $\partial_{[\mu} e(x) \partial_{\nu]} f=0, \forall f \in C^{1}\left(\mathbb{R}^{D}\right)[6]$. This is not however a sufficient condition. The associativity of the twisted star product implies the Jacobi identity

$$
J(\mu, \nu, \rho)=\left[x^{\mu},\left[x^{\nu}, x^{\rho}\right]_{\star}\right]_{\star}+\left[x^{\rho},\left[x^{\mu}, x^{\nu}\right]_{\star}\right]_{\star}+\left[x^{\nu},\left[x^{\rho}, x^{\mu}\right]_{\star}\right]_{\star}=0 .
$$

The particular case of the structure function

$$
e(x)=1+\omega_{\mu} x^{\mu}
$$

where $\omega_{\mu} x^{\mu}$ is dimensionless and $\omega_{\mu} \in \mathbb{R}$, leads to

$$
J(\mu, \nu, \rho)=-e(x) \omega_{\sigma}\left(\theta^{\nu \rho} \theta^{\mu \sigma}+\theta^{\mu \nu} \theta^{\rho \sigma}+\theta^{\rho \mu} \theta^{\nu \sigma}\right) .
$$

For such a choice of the function $e(x)$, the associativity of the star product (5) can be shown even for the non-vanishing tensor $\omega_{\sigma}$ [8]. From this point, the authors of [8] were able to derive the equivalent of the so-called matrix basis of the Moyal plane [10, 9].

$\mathrm{NC}$ spaces can be slightly more general than the above. For instance there are several developments around the so called NC quantum mechanics [13, 18, 19, 20, 21. NC quantum mechanics [20] can be also described by introducing commutation relations between coordinate and momentum even also between momentum and momentum. Thus (6) can be extended to a $2 D \mathrm{NC}$ phase space as follows

$$
\left[x^{1}, x^{2}\right]_{\star}=i \theta e(x), \quad\left[x^{1}, p^{1}\right]_{\star}=i \hbar_{e f f}, \quad\left[x^{2}, p^{2}\right]_{\star}=i \hbar_{e f f}, \quad\left[p^{1}, p^{2}\right]=i \bar{\theta}
$$


where $\theta, \bar{\theta}$ and $\hbar_{e f f}$ are constant but $e(x)=1+\omega_{1} x^{1}+\omega_{2} x^{2}$ is still a function. The present work highlights the spectrum of the harmonic oscillator in this twisted NC phase space defined by the commutation relations (10), with the restriction $\omega_{1}=0$. We show, using a particular transformation of the basic degrees of freedom that the total nonlinear harmonic oscillator Hamiltonian factorizes. From that point, the model becomes solvable.

The paper is organized as follows. In section 2 , we give some useful results concerning the deformation of the NC phase space (10). Then, the spectrum and states of the harmonic oscillator are solved. We give a summary of our results in section 3 .

\section{Position-dependent NC quantum mechanics}

This section addresses the construction of a position-dependent NC star product which is induced by the deformation (10). We start with the following definition.

Definition 1 (Twisted Moyal algebra). Consider the set $E=\left\{\left(x^{i}, p^{i}\right), i=1,2\right\}$ and $\mathbb{C}\left[\left[x^{1}, x^{2}, p^{1}, p^{2}\right]\right]$, the free algebra generated by $E$. Let $\mathcal{I}$ be the ideal of $\mathbb{C}\left[\left[x^{1}, x^{2}, p^{1}, p^{2}\right]\right]$, generated by the elements $\left[x^{i}, x^{j}\right]_{\star}-i \theta^{i j}(x), \quad\left[x^{i}, p^{j}\right]_{\star}-i \hbar_{e f f} \delta^{i j}, \quad\left[p^{i}, p^{j}\right]_{\star}-i \bar{\theta}^{i j}$, where $\theta^{i j}(x)$ is skew symmetric tensor depending on space coordinates and $\bar{\theta}^{i j}$ a constant skew symmetric tensor. The twisted Moyal algebra $\mathcal{M}_{\theta \bar{\theta} \hbar_{e f f}}$ is the quotient $\mathbb{C}\left[\left[x^{1}, x^{2}, p^{1}, p^{2}\right]\right] / \mathcal{I}$. Each element in $\mathcal{M}_{\theta \bar{\theta} \hbar_{e f f}}$ is a formal power series in the $\left(x^{i}, p^{j}\right)$ 's for which the following relations hold:

$$
\left[x^{i}, x^{j}\right]_{\star}=i \theta^{i j}(x), \quad\left[x^{i}, p^{j}\right]_{\star}=i \hbar_{e f f} \delta^{i j}, \quad\left[p^{i}, p^{j}\right]_{\star}=i \bar{\theta}^{i j} .
$$

The Moyal algebra can be also defined as the linear space of smooth and rapidly decreasing functions equipped with the NC star product given in the form $f \star g=\mathbf{m}\left[\left(\star_{\theta} \star_{\hbar_{e f f}} \star_{\bar{\theta}}\right)(f \otimes g)\right]$, such that

$$
\begin{aligned}
& f \star_{\theta} g=\mathbf{m}\left[\exp \left(\frac{i}{2} \theta^{i j}(x) \partial_{x^{i}} \otimes \partial_{x^{j}}\right) f \otimes g\right] \\
& f \star_{\hbar_{e f f}} g=\mathbf{m}\left[\exp \left(\frac{i}{2} \hbar_{e f f} \delta^{i j}\left(\partial_{x^{i}} \otimes \partial_{p^{j}}-\partial_{p^{i}} \otimes \partial_{x^{j}}\right)\right) f \otimes g\right] \\
& f \star_{\bar{\theta}} g=\mathbf{m}\left[\exp \left(\frac{i}{2} \bar{\theta}^{i j} \partial_{p^{i}} \otimes \partial_{p^{j}}\right) f \otimes g\right] .
\end{aligned}
$$

For $D=2$, we set $x^{i}=\left(x^{1}, x^{2}\right), p^{i}=\left(p^{1}, p^{2}\right)$ with $\left(x^{i}, p^{i}\right) \in \mathbb{R}^{4}$ and we will restrict the NC structure tensors to the following:

$$
\theta^{i j}(x)=\theta e(x)\left(\begin{array}{cc}
0 & 1 \\
-1 & 0
\end{array}\right)=\theta e(x) \epsilon^{i j}, \quad \bar{\theta}^{i j}=\bar{\theta}\left(\begin{array}{cc}
0 & 1 \\
-1 & 0
\end{array}\right)=\bar{\theta} \epsilon^{i j} .
$$

For $f \in C^{\infty}\left(\mathbb{R}^{4}\right)$, the following relations are satisfied

$$
\begin{array}{ll}
x^{1} \star f=x^{1} f+\frac{i \theta e}{2} \partial_{x^{2}} f+\frac{i \hbar_{e f f}}{2} \partial_{p^{1}} f, & p^{1} \star f=p^{1} f+\frac{i \bar{\theta}}{2} \partial_{p^{2}} f-\frac{i \hbar_{e f f}}{2} \partial_{x^{1}} f, \\
x^{2} \star f=x^{2} f-\frac{i \theta e}{2} \partial_{x^{1}} f+\frac{i \hbar_{e f f}}{2} \partial_{p^{2}} f, & p^{2} \star f=p^{2} f-\frac{i \bar{\theta}}{2} \partial_{p^{1}} f-\frac{i \hbar_{e f f}}{2} \partial_{x^{2}} f .
\end{array}
$$

The commutation relation (11) can be deduced from (16) and (17). 
We can now introduce a model on that twisted $\mathrm{NC}$ space. Let us consider the NC harmonic oscillator described by the Hamiltonian

$$
H=\frac{1}{2}\left[\left(p^{1}\right)^{2}+\left(p^{2}\right)^{2}+\left(x^{1}\right)^{2}+\left(x^{2}\right)^{2}\right] .
$$

In (18), the mass parameter and the oscillator constant are taken to be 1 . The Hamiltonian (18) is invariant under the phase space rotation. We now address the problem we want to solve.

We will solve the eigenvalue problem associated with (18) in the NC phase space

$$
H \star \psi=E \psi .
$$

A way to solve the eigenvalue problem of a quantum Hamiltonian is its factorization. In the following, will introduce a particular type of factorization. The eigenvalue problem (19) can be split into two equations given by

$$
H_{R} \star \psi=E \psi, \quad \text { and } \quad H_{I m} \star \psi=0
$$

where, by expansion of the twisted star product, one should obtain the real and imaginary part corresponding to (19) as:

$$
\begin{aligned}
H_{R} & =\frac{1}{2}\left[\left(p^{1}\right)^{2}+\left(p^{2}\right)^{2}+\left(x^{1}\right)^{2}+\left(x^{2}\right)^{2}++\frac{\bar{\theta} \hbar_{e f f}}{2}\left(\partial_{p^{2}} \partial_{x^{1}}-\partial_{p^{1}} \partial_{x^{2}}\right)\right. \\
& -\frac{\theta \hbar_{e f f} e}{2}\left(\partial_{x^{2}} \partial_{p^{1}}-\partial_{x^{1}} \partial_{p^{2}}\right)-\frac{\hbar_{e f f}^{2}}{4}\left(\partial_{p^{1}}^{2}+\partial_{p^{2}}^{2}+\partial_{x^{1}}^{2}+\partial_{x^{2}}^{2}\right) \\
& \left.-\frac{\bar{\theta}^{2}}{4}\left(\partial_{p^{1}}^{2}+\partial_{p^{2}}^{2}\right)-\frac{\theta^{2} e}{4}\left(\partial_{x^{1}}^{2}+\partial_{x^{2}}^{2}\right)-\frac{\theta e}{4}\left(\omega_{2} \partial_{x^{2}}+\omega_{1} \partial_{x^{1}}\right)\right]
\end{aligned}
$$

and

$$
H_{I m}=\bar{\theta}\left(p^{1} \partial_{p^{2}}-p^{2} \partial_{p^{1}}\right)+\theta e\left(x^{1} \partial_{x^{2}}-x^{2} \partial_{x^{1}}\right)+\hbar_{e f f}\left(x^{1} \partial_{p^{1}}+x^{2} \partial_{p^{2}}-p^{1} \partial_{x^{1}}-p^{2} \partial_{x^{2}}\right) .
$$

Note that the eigenvalue equation (19) can be also written as $\psi \star H=E \psi$, due to the fact that $f \star g=\overline{g \star f}$. Equations (20) with the Hamiltonians (21) and (22) are nonlinear. However after putting a restriction on the type of noncommutativity that we use, we will provide solution to these equations.

Solution - Consider $e(x)=1+\omega_{1} x^{1}+\omega_{2} x^{2}$. Let us assume that $\omega_{1} \neq 0$ or $\omega_{2} \neq 0$. Consider the transformation $\mathcal{T}$ mapping coordinates $(x, p)$ to the new variables $(\widetilde{x}, \widetilde{p})$ given by

$$
\mathcal{T}:\left\{\begin{array}{c}
x^{1}=\theta \omega_{1} e^{\widetilde{x}^{1}}-\theta \omega_{2} \widetilde{x}^{2}-\frac{\omega_{1}}{\omega_{1}^{2}+\omega_{2}^{2}} \\
x^{2}=\theta \omega_{2} e^{\widetilde{x}^{1}}+\theta \omega_{1} \widetilde{x}^{2}-\frac{\omega_{2}}{\omega_{1}^{2}+\omega_{2}^{2}} \\
p^{1}=\widetilde{p}^{1} \\
p^{2}=\widetilde{p}^{2}
\end{array}\right.
$$

For $\theta>0$, the transformation $\mathcal{T}$ is invertible in the positive domain of the plane $\left(x^{1}, x^{2}\right)$ given by relation

$$
e(x)=1+\omega_{1} x^{1}+\omega_{2} x^{2}>0
$$


The inverse transformation $\mathcal{T}^{-1}$ is given by

$$
\mathcal{T}^{-1}:\left\{\begin{array}{c}
\widetilde{x}^{1}=\ln \left(\frac{e(x)}{\theta\left(\omega_{1}^{2}+\omega_{2}^{2}\right)}\right) \\
\widetilde{x}^{2}=\frac{-\omega_{2} x^{1}+\omega_{1} x^{2}}{\theta\left(\omega_{1}^{2}+\omega_{2}^{2}\right)} \\
\widetilde{p}^{1}=p^{1} \\
\widetilde{p}^{2}=p^{2}
\end{array}\right.
$$

Let us immediately remark that the transformation (25) break the ordinary limit of the theory i.e. the limit $\theta \rightarrow 0$ cannot be taken into account. To recover this inconvenience we can use the renormalization procedures, which will be addressed in forthcoming work.

Under $\mathcal{T}$, the algebra $\mathcal{M}_{\theta \bar{\theta} \hbar_{e f f}}$ is transformed as $\widetilde{\mathcal{M}}_{\theta \bar{\theta} \hbar_{e f f}}=\mathcal{T}\left[\mathcal{M}_{\theta \bar{\theta} \hbar_{e f f}}\right]$. The nonvanishing commutation relations satisfied by the new variables are given by the following:

$$
\begin{aligned}
& {\left[\widetilde{x}^{1}, \widetilde{x}^{2}\right]_{\star}=i \theta \sqrt{\omega_{1}^{2}+\omega_{2}^{2}}=i \gamma, \quad\left[\widetilde{x}^{1}, \widetilde{p}^{1}\right]_{\star}=i \hbar_{e f f} \omega_{1} e^{-1}=i \hbar_{1}(x),} \\
& {\left[\widetilde{x}^{2}, \widetilde{p}^{2}\right]_{\star}=i \frac{\omega_{1} \hbar_{e f f}}{\theta\left(\omega_{1}^{2}+\omega_{2}^{2}\right)}=i \hbar_{2}, \quad\left[\widetilde{p}^{1}, \widetilde{p}^{2}\right]_{\star}=i \bar{\theta} .}
\end{aligned}
$$

The ordinary recipes that are known for diagonalizing the algebra do not work in this instance because of the presence of the function $e(x)$. However, restricting to the case $\omega_{1}=0$ and $\omega_{2} \neq 0$, we have

$$
x^{1}=-\gamma \widetilde{x}^{2}, \quad x^{2}=\gamma e^{\widetilde{x}^{1}}-\frac{1}{\omega_{2}}, \quad p^{1}=\widetilde{p}^{1}, \quad p^{2}=\widetilde{p}^{2}, \quad \gamma=\theta \omega_{2} .
$$

Therefore, for simplicity by setting $\omega_{1}=0$ and $\omega_{2}=1=\gamma$, we understand that the transformation $\mathcal{T}$ simply induces a rotation in the plane $\left(x^{1}, x^{2}\right) \rightarrow\left(x^{2},-x^{1}\right)$ followed by a logarithmic scale transformation $\left(x^{1}, x^{2}\right) \rightarrow\left(\ln \left[x^{1}+1\right], x^{2}\right)$. Note that such a transformation cannot be defined in the case of the Moyal plane determined by the limiting situation $\omega_{1}=$ $\omega_{2}=0$. Furthermore, it can be noticed that the restriction (28) clearly breaks the symmetry between the coordinates $x^{1}$ and $x^{2}$. In any case, the following analysis finds an analog when we consider $\omega_{2}=0, \omega_{1} \neq 0$.

We obtain the final commutation relations

$$
\left[\widetilde{x}^{1}, \widetilde{p}^{1}\right]_{\star}=0, \quad\left[\widetilde{x}^{2}, \widetilde{p}^{2}\right]_{\star}=0, \quad\left[\widetilde{x}^{1}, \widetilde{x}^{2}\right]_{\star}=i \gamma, \quad\left[\widetilde{p}^{1}, \widetilde{p}^{2}\right]_{\star}=i \bar{\theta}
$$

As a consequence, the algebra $\widetilde{\mathcal{M}}_{\theta \bar{\theta} \hbar_{e f f}}$ splits into two sectors $\widetilde{\mathcal{M}}_{\theta}$ and $\widetilde{\mathcal{M}}_{\bar{\theta}}$ such that

$$
\widetilde{\mathcal{M}}_{\theta} \otimes \widetilde{\mathcal{M}}_{\bar{\theta}} \equiv \widetilde{\mathcal{M}}_{\theta \bar{\theta} \hbar_{e f f}}
$$

where the algebras $\widetilde{\mathcal{M}}_{\theta}$ and $\widetilde{\mathcal{M}}_{\bar{\theta}}$ are each of the Moyal-type defined such that $\widetilde{\mathcal{M}}_{\theta}=$ $\mathbb{C}\left[\left[\widetilde{x}^{1}, \widetilde{x}^{2}\right]\right] / \mathcal{I}_{1}$ and $\widetilde{\mathcal{M}}_{\bar{\theta}}=\mathbb{C}\left[\left[\widetilde{p}^{1}, \widetilde{p}^{2}\right]\right] / \mathcal{I}_{2}$, where $\mathcal{I}_{1}$ and $\mathcal{I}_{2}$ are, respectively, the ideal of $\mathbb{C}\left[\left[\widetilde{x}^{1}, \widetilde{x}^{2}\right]\right]$, generated by the elements $\left[\widetilde{x}^{1}, \widetilde{x}^{2}\right]_{\star}-i \gamma$, and the ideal of $\mathbb{C}\left[\left[\widetilde{p}^{1}, \widetilde{p}^{2}\right]\right]$, generated by the elements $\left[\widetilde{p}^{1}, \widetilde{p}^{2}\right]_{\star}-i \bar{\theta}$. In short, $\widetilde{\mathcal{M}}_{\theta} \otimes \widetilde{\mathcal{M}}_{\bar{\theta}}$ defines a standard 4 dimensional Moyal space $\left[y^{\alpha}, y^{\beta}\right]=\Theta^{\alpha \beta}, \alpha, \beta=1,2,3,4$, with tensor structure

$$
\Theta:=\left(\begin{array}{cc}
\gamma J & 0 \\
0 & \theta J
\end{array}\right) \quad J:=\left(\begin{array}{cc}
0 & 1 \\
-1 & 0
\end{array}\right)
$$


where $y^{1,2}=\widetilde{x}^{1,2}$ and $y^{3,4}=\widetilde{p}^{1,2}$.

For simplicity, we set $\gamma=1, \omega_{2}=2$ and $\bar{\theta}=1$. Using (28), the Hamiltonian (18) takes the form

$$
H=\frac{1}{2}\left[\gamma^{2}\left(\widetilde{x}^{2}\right)^{2}+\gamma^{2} e^{2 \widetilde{x}^{1}}-\frac{2 \gamma}{\omega_{2}} e^{\widetilde{x}^{1}}+\frac{1}{\omega_{2}^{2}}+\left(\widetilde{p}^{1}\right)^{2}+\left(\widetilde{p}^{2}\right)^{2}\right]=H_{1}\left(\widetilde{x}^{1}, \widetilde{x}^{2}\right)+H_{2}\left(\widetilde{p}^{1}, \widetilde{p}^{2}\right)
$$

where

$$
H_{1}\left(\widetilde{x}^{1}, \widetilde{x}^{2}\right)=\frac{1}{2}\left[\left(\widetilde{x}^{2}\right)^{2}+e^{2 \widetilde{x}^{1}}-e^{\widetilde{x}^{1}}+\frac{1}{4}\right], \quad H_{2}\left(\widetilde{p}^{1}, \widetilde{p}^{2}\right)=\frac{1}{2}\left[\left(\widetilde{p}^{1}\right)^{2}+\left(\widetilde{p}^{2}\right)^{2}\right] .
$$

Now using the commutation relations (29), we get

$$
\left[H_{1}\left(\widetilde{x}^{1}, \widetilde{x}^{2}\right), H_{2}\left(\widetilde{p}^{1}, \widetilde{p}^{2}\right)\right]_{\star}=0 .
$$

It appears clear that the star product $\star=\star_{\theta} \star_{\hbar_{e f f}} \star_{\bar{\theta}}$ gets mapped as

$$
\star \longrightarrow \mathcal{T}(\star)=\star_{1} \star_{2}
$$

with

$$
\star_{1}=\mathbf{m}\left[\exp \left(\frac{i}{2}\left(\partial_{\widetilde{x}^{1}} \otimes \partial_{\widetilde{x}^{2}}-\partial_{\widetilde{x}^{2}} \otimes \partial_{\widetilde{x}^{1}}\right)\right)\right], \quad \star_{2}=\mathbf{m}\left[\exp \left(\frac{i}{2}\left(\partial_{\widetilde{p}^{1}} \otimes \partial_{\widetilde{p}^{2}}-\partial_{\widetilde{p}^{2}} \otimes \partial_{\widetilde{p}^{1}}\right)\right)\right]
$$

Then, the new coordinate and momentum operators can be described by the following relations

$$
\widetilde{x}^{1} \star_{1}=\widetilde{x}^{1}+\frac{i}{2} \partial_{\widetilde{x}^{2}}, \quad \widetilde{x}^{2} \star_{1}=\widetilde{x}^{2}-\frac{i}{2} \partial_{\widetilde{x}^{1}}, \quad \widetilde{p}^{1} \star_{2}=\widetilde{p}^{1}+\frac{i}{2} \partial_{\widetilde{p}^{2}}, \quad \widetilde{p}^{2} \star_{2}=\widetilde{p}^{2}-\frac{i}{2} \partial_{\widetilde{p}^{1}} .
$$

The initial Hamiltonian has been factorized into two commuting sectors. We can first study the spectrum of Hamiltonian $H_{1}\left(\widetilde{x}^{1}, \widetilde{x}^{2}\right)$ so called supersymmetric Liouville Hamiltonian. Using (37), the Hamiltonian in this sector takes the form

$$
\begin{aligned}
H_{1}\left(\widetilde{x}^{1}, \widetilde{x}^{2}\right) \star_{1} & =\frac{1}{2}\left[\left(\widetilde{x}^{2}\right)^{2}-i \widetilde{x}^{2} \partial_{\widetilde{x}^{1}}-\frac{1}{4} \partial_{\widetilde{x}^{1}}^{2}+e^{2 \widetilde{x}^{1}}\left(\cos \partial_{\widetilde{x}^{2}}+i \sin \partial_{\widetilde{x}^{2}}\right)\right. \\
& \left.-e^{\widetilde{x}^{1}}\left(\cos \frac{1}{2} \partial_{\widetilde{x}^{2}}+i \sin \frac{1}{2} \partial_{\widetilde{x}^{2}}\right)+\frac{1}{4}\right]
\end{aligned}
$$

For a real $E_{1}$ and a wave function $\psi_{1, E_{1}}$, the eigenvalue problem $H_{1}\left(\widetilde{x}^{1}, \widetilde{x}^{2}\right) \star_{1} \psi_{1, E_{1}}=E_{1} \psi_{1, E_{1}}$ can be re-expressed into two parts: the real part is given by

$$
\left(\left(\widetilde{x}^{2}\right)^{2}-\frac{1}{4} \partial_{\widetilde{x}^{1}}^{2}+e^{2 \widetilde{x}^{1}} \cos \partial_{\widetilde{x}^{2}}-e^{\widetilde{x}^{1}} \cos \frac{1}{2} \partial_{\widetilde{x}^{2}}+\frac{1}{4}-2 E_{1}\right) \psi_{1, E_{1}}=0
$$

whereas the imaginary part expresses as

$$
\left(\widetilde{x}^{2} \partial_{\widetilde{x}^{1}}-e^{2 \widetilde{x}^{1}} \sin \partial_{\widetilde{x}^{2}}+e^{\widetilde{x}^{1}} \sin \frac{1}{2} \partial_{\widetilde{x}^{2}}\right) \psi_{1, E_{1}}=0 .
$$


To solve consistently the equations (39) and (40), we will use a fact about the Taylor expansion of an arbitrary function $\psi(x)$, for the small values of parameter $\epsilon$ as

$$
\begin{gathered}
\psi(x+\epsilon)=\psi(x)+\epsilon \partial_{x} \psi(x)+\frac{1}{2} \epsilon^{2} \partial_{x}^{2} \psi(x)+\cdots=e^{\epsilon \partial_{x}} \psi(x) \\
\psi(x-\epsilon)=\psi(x)-\epsilon \partial_{x} \psi(x)+\frac{1}{2} \epsilon^{2} \partial_{x}^{2} \psi(x)+\cdots=e^{-\epsilon \partial_{x}} \psi(x) .
\end{gathered}
$$

Then summing (41) and (42), we get

$$
\begin{aligned}
& \frac{1}{2}(\psi(x+\epsilon)+\psi(x-\epsilon))=\cosh \epsilon \partial_{x} \psi(x), \\
& \frac{1}{2}(\psi(x+\epsilon)-\psi(x-\epsilon))=\sinh \epsilon \partial_{x} \psi(x) .
\end{aligned}
$$

We restrict to the case where $\epsilon=i$ and $\epsilon=\frac{i}{2}$. Then follow from the identities

$$
\begin{aligned}
& \left(\sin \partial_{\widetilde{x}^{2}}\right) \psi\left(\widetilde{x}^{1}, \widetilde{x}^{2}\right)=\frac{1}{2 i}\left(\psi\left(\widetilde{x}^{1}, \widetilde{x}^{2}+i\right)-\psi\left(\widetilde{x}^{1}, \widetilde{x}^{2}-i\right)\right) \\
& \left(\cos \partial_{\widetilde{x}^{2}}\right) \psi\left(\widetilde{x}^{1}, \widetilde{x}^{2}\right)=\frac{1}{2}\left(\psi\left(\widetilde{x}^{1}, \widetilde{x}^{2}+i\right)+\psi\left(\widetilde{x}^{1}, \widetilde{x}^{2}-i\right)\right)
\end{aligned}
$$

and

$$
\begin{aligned}
& \left(\sin \frac{1}{2} \partial_{\widetilde{x}^{2}}\right) \psi\left(\widetilde{x}^{1}, \widetilde{x}^{2}\right)=\frac{1}{2 i}\left(\psi\left(\widetilde{x}^{1}, \widetilde{x}^{2}+i / 2\right)-\psi\left(\widetilde{x}^{1}, \widetilde{x}^{2}-i / 2\right)\right) \\
& \left(\cos \frac{1}{2} \partial_{\widetilde{x}^{2}}\right) \psi\left(\widetilde{x}^{1}, \widetilde{x}^{2}\right)=\frac{1}{2}\left(\psi\left(\widetilde{x}^{1}, \widetilde{x}^{2}+i / 2\right)+\psi\left(\widetilde{x}^{1}, \widetilde{x}^{2}-i / 2\right)\right) .
\end{aligned}
$$

The equation (40) can be simply written as

$$
\begin{aligned}
\left(\widetilde{x}^{2} \partial_{\widetilde{x}^{1}}\right) \psi_{1, E}\left(\widetilde{x}^{1}, \widetilde{x}^{2}\right) & =\frac{e^{2 \widetilde{x}^{1}}}{2 i}\left(\psi_{1, E}\left(\widetilde{x}^{1}, \widetilde{x}^{2}+i\right)-\psi_{1, E}\left(\widetilde{x}^{1}, \widetilde{x}^{2}-i\right)\right) \\
& -\frac{e^{\widetilde{x}^{1}}}{2 i}\left(\psi\left(\widetilde{x}^{1}, \widetilde{x}^{2}+i / 2\right)-\psi\left(\widetilde{x}^{1}, \widetilde{x}^{2}-i / 2\right)\right) .
\end{aligned}
$$

Using the above relations, we then get the new equation corresponding to (39) as

$$
\begin{aligned}
& \left(\left(\widetilde{x}^{2}\right)^{2}+\frac{1}{4}-2 E_{1}\right) \psi_{1, E_{1}}\left(\widetilde{x}^{1}, \widetilde{x}^{2}\right)-\frac{1}{4}\left[\frac{e^{2 \widetilde{x}^{1}}}{i \widetilde{x}^{2}}\left(\psi_{1, E}\left(\widetilde{x}^{1}, \widetilde{x}^{2}+i\right)-\psi_{1, E}\left(\widetilde{x}^{1}, \widetilde{x}^{2}-i\right)\right)\right. \\
& -\frac{e^{\widetilde{x}^{1}}}{2 i \widetilde{x}^{2}}\left(\psi\left(\widetilde{x}^{1}, \widetilde{x}^{2}+i / 2\right)-\psi\left(\widetilde{x}^{1}, \widetilde{x}^{2}-i / 2\right)\right)-\frac{e^{4 \widetilde{x}^{1}}}{4\left(\widetilde{x}^{2}\right)^{2}}\left(\psi\left(\widetilde{x}^{1}, \widetilde{x}^{2}+2 i\right)-\psi\left(\widetilde{x}^{1}, \widetilde{x}^{2}\right)\right) \\
& \left.+\frac{e^{2 \widetilde{x}^{1}}}{4\left(\widetilde{x}^{2}\right)^{2}}\left(\psi\left(\widetilde{x}^{1}, \widetilde{x}^{2}+i\right)-\psi\left(\widetilde{x}^{1}, \widetilde{x}^{2}\right)+2 \psi\left(\widetilde{x}^{1}, \widetilde{x}^{2}-i\right)\right)\right] \\
& \left.-\frac{e^{2 \widetilde{x}^{1}}}{2}\left(\psi\left(\widetilde{x}^{1}, \widetilde{x}^{2}+i\right)+\psi\left(\widetilde{x}^{1}, \widetilde{x}^{2}-i\right)\right)+\frac{e^{\widetilde{x}^{1}}}{2}\left(\psi\left(\widetilde{x}^{1}, \widetilde{x}^{2}+i / 2\right)+\psi\left(\widetilde{x}^{1}, \widetilde{x}^{2}-i / 2\right)\right)\right]=0 .
\end{aligned}
$$

In [14, the recursive properties of the Meijer G-function can be used to compute the eigenvectors $\psi_{1, E_{1}}\left(\widetilde{x}^{1}\right)$ as

$$
\psi_{1, E_{1}}\left(\widetilde{x}^{1}\right)=\left(\frac{1}{4 \pi^{2} \sqrt{E_{1}}} e^{\widetilde{x}^{1}} \cosh \left(\pi \sqrt{E_{1}}\right)\right)^{1 / 2}\left(K_{\frac{1}{2}-i \sqrt{E_{1}}}\left(e^{\widetilde{x}^{1}}\right)+K_{\frac{1}{2}+i \sqrt{E_{1}}}\left(e^{\widetilde{x}^{1}}\right)\right), E_{1} \geq 0
$$


where $K$ are Kelvin (modified Bessel) functions.

The second eigenvalue problem $H_{2}\left(\widetilde{p}^{1}, \widetilde{p}^{2}\right) \star_{2} \psi_{2, E_{2}}=E_{2} \psi_{2, E_{2}}$ is well known as the simple quantum harmonic oscillator problem. We write

$$
\psi_{2, E_{2}}\left(\widetilde{p}^{1}\right)=\left(\frac{1}{\pi}\right)^{1 / 4} \frac{1}{2^{n} n !} H_{n}\left(\widetilde{p}^{1}\right) e^{-\left(\widetilde{p}^{1}\right)^{2} / 2}
$$

where $H_{n}$ stand for the Hermite polynomial and the oscillator energy as $E_{2}=n+\frac{1}{2}$. Finally the solution of Hamiltonian (18) is then

$$
\psi_{E}\left(\widetilde{x}^{1}, \widetilde{p}^{1}\right)=\psi_{1, E_{1}}\left(\widetilde{x}^{1}\right) \otimes \psi_{2, E_{2}}\left(\widetilde{p}^{1}\right), \quad E=E_{1}+E_{2} .
$$

We conclude that the spectrum of the Hamiltonian $H$ is composed by two sectors: A continuum part in the sector $H_{1}$ and a discrete one in the sector $H_{2}$.

\section{Conclusion}

In this work, following our previous approach [7] and results based on [14], we have found the eigenvalues and eigenvectors of the harmonic oscillator in the twisted Moyal space with function structure $e(x)=1+\omega_{2} x^{2}$. We have introduced a particular transformation which has allowed us to split the total twisted Moyal algebra into two parts in which the Hamiltonian was written in two commuting pieces.

Let us remark that the solution (53) exhibits the lack of commutative limit $\theta \rightarrow 0$. This inconvenience is due to the form of the scale transformation $\mathcal{T}$. Therefore the solution

obtained in relation (51) need to be renormalized. Note also that it is a more difficult problem to find a solution for the harmonic oscillator in the more symmetric case of $e(x)=1+\omega_{\mu} x^{\mu}$. This two tangles deserves to be investigated.

\section{Acknowledgements}

The author would like to thank Joseph Ben Geloun for useful comments which have improved this work. This research was supported in part by Perimeter Institute for Theoretical Physics. Research at Perimeter Institute is supported by the Government of Canada through Industry Canada and by the Province of Ontario through the Ministry of Research and Innovation.

\section{References}

[1] Y. S. Kim and E. P. Wigner, "Covariant Phase Space Representation For Harmonic Oscillators," Phys. Rev. A 38, 1159 (1988).

[2] R. J. Szabo, "Quantum field theory on noncommutative spaces," Phys. Rept. 378, 207 (2003) hep-th/0109162].

[3] J. A. Crawford, "A noncommutative representation of classical dynamics. connections with field quantization," Nuovo Cim. B 9, 1 (1972). 
[4] A. Kempf and G. Mangano, "Minimal length uncertainty relation and ultraviolet regularization," Phys. Rev. D 55, 7909 (1997) hep-th/9612084.

[5] D. Sternheimer, "Deformation quantization: Twenty years after," AIP Conf. Proc. 453, 107 (1998) math/9809056.

[6] P. Aschieri, L. Castellani and M. Dimitrijevic, "Dynamical noncommutativity and Noether theorem in twisted phi***4 theory," Lett. Math. Phys. 85, 39 (2008) arXiv:0803.4325 [hep-th]].

[7] M. N. Hounkonnou and D. O. Samary, "Harmonic oscillator in twisted Moyal plane: eigenvalue problem and relevant properties," J. Math. Phys. 51, 102108 (2010) arXiv:1008.1325 [math-ph]].

[8] M. N. Hounkonnou and D. O. Samary, "Twisted Yang-Mills field theory: connections and Noether currents," J. Phys. A 44, 315401 (2011).

[9] A. de Goursac, J. -C. Wallet and R. Wulkenhaar, "On the vacuum states for noncommutative gauge theory," Eur. Phys. J. C 56, 293 (2008) [arXiv:0803.3035 [hep-th]].

[10] H. Grosse and R. Wulkenhaar, "Renormalization of phi**4 theory on noncommutative $\mathrm{R}^{* *} 2$ in the matrix base," JHEP 0312, 019 (2003) hep-th/0307017].

[11] P. Valtancoli, "Algebraic method for the harmonic oscillator with a minimal length," arXiv:1306.0117 [hep-th].

[12] F. Benatti and L. Gouba, "Classical limits of quantum mechanics on a non-commutative configuration space," J. Math. Phys. 54, 063508 (2013) [arXiv:1302.4284 [math-ph]].

[13] X. -F. Diao, G. -J. Guo and Z. -W. Long, "The eigenvalues of two modes coupled harmonic oscillators in noncommutative phase-space," Int. J. Mod. Phys. A 26, 1561 (2011).

[14] T. Curtright, D. Fairlie and C. K. Zachos, "Features of time independent Wigner functions," Phys. Rev. D 58, 025002 (1998) hep-th/9711183.

[15] S. Dey, A. Fring and B. Khantoul, "Hermitian versus non-Hermitian representations for minimal length uncertainty relations," arXiv:1302.4571 [quant-ph].

[16] S. Dey and A. Fring, "The two dimensional harmonic oscillator on a noncommutative space with minimal uncertainties," Acta Polytechnica 53, 268 (2013) arXiv:1207.3303 [hep-th]].

[17] P. G. Castro, B. Chakraborty, R. Kullock and F. Toppan, "Noncommutative Oscillators from a Hopf Algebra Twist Deformation. A first Principles Derivation," J. Math. Phys. 52, 032102 (2011) arXiv:1012.5158 [hep-th]].

[18] J. Wang, K. Li and S. Dulat, "Wigner Functions for harmonic oscillator in noncommutative phase space," arXiv:0908.1703 [hep-th]. 
[19] J. Ben Geloun, S. Gangopadhyay and F. G Scholtz, "Harmonic oscillator in a background magnetic field in noncommutative quantum phase-space," Europhys. Lett. 86, 51001 (2009) [arXiv:0901.3412 [hep-th]].

[20] F. G. Scholtz, L. Gouba, A. Hafver and C. M. Rohwer, "Formulation, Interpretation and Application of non-Commutative Quantum Mechanics," J. Phys. A 42, 175303 (2009) arXiv:0812.2803 [math-ph]].

[21] C. M. Rohwer, K. G. Zloshchastiev, L. Gouba and F. G. Scholtz, "Noncommutative quantum mechanics: A Perspective on structure and spatial extent," J. Phys. A 43, 345302 (2010) arXiv:1004.1984 [math-ph]].

[22] J. Jing, S. -H. Zhao, J. -F. Chen and Z. -W. Long, "On the spectra of noncommutative 2D harmonic oscillator," Eur. Phys. J. C 54, 685 (2008). 